\title{
Les intellectuelles et l'amour : Marie de Gournay et Marguerite de Valois
}

RICHARD HILLMAN

Summary: When a woman defined herself as an intellectual in the Renaissance, she could not, even if she wished, detach that role from the cultural expectations conventionally attached to her gender, and most notably from issues of love and sexuality. This point may be illustrated with reference to the attempts of Marie de Gournay and Marguerite de Valois to manage by intellectual means the discourses surrounding their personal lives. Despite the contrasting stereotypes ultimately imposed upon them - "old maid" in Gournay's case, "whore” in Marguerite's - the recourse of both women to versions of neo-platonic philosophy is ultimately inseparable from the erotic discourses in which they found themselves pre-inscribed.

T e but principal de cet article est de distinguer les parcours de deux Lintellectuelles à l'égard de leur féminité, et de le faire à travers les discours qui leur étaient consacrés ; il convient alors de commencer par un point de contact biographique, qui est aussi un point de contraste . Il est bien connu que Marie de Gournay fréquentait le salon présidé par l'ancienne reine de France entre le retour spectaculaire à Paris de cette dernière en 1605 et sa mort en 1615. Éliane Viennot accorde à Marie de Gournay une place certaine dans l' « entourage » de Marguerite de Valois, qui, d'après des documents, fournissait à la femme écrivain une aide financière ${ }^{1}$.

Toutefois Marjorie Henry Ilsley a sans doute tendance à idéaliser cette relation, lorsqu'elle comble le manque d'information en esquissant une véritable intimité entre intellectuelles ${ }^{2}$. Il est évident que les deux femmes restaient séparées par un gouffre social et économique : le rôle important joué par Marguerite, même divorcée, au sein de la vie publique, courtoise et culturelle manifestait son pouvoir social découlant du sang royal, à la différence de l'impuissance et précarité de Gournay, qui, paradoxalement, 
fut toujours dépendante de la faveur des autres pour maintenir son indépendance. La quasi-misère que Gournay évoque si vivement dans son Apologie pour celle qui écrit aurait dû trancher avec la vie fastueuse menée par Marguerite. Pourtant, à part leurs goûts intellectuels, les deux femmes avaient certainement des choses en commun, y compris l'obligation, inhérente au rôle de femme intellectuelle dans un milieu profondément misogyne, de gérer publiquement leur vie privée, et particulièrement cet aspect de la vie que l'on peut étiqueter comme « amoureux ». Voilà l'idée centrale du présent article, qui prendra donc comme point de départ, mais dans un esprit plutôt sceptique, un des liens évoqués par Ilsley imaginant les discussions dans le salon : «One of the subjects frequently proposed by Marguerite was that of love about which she had, like Marie de Gournay, modern ideas $»^{3}$.

Il n'est guère surprenant qu'une femme de l'époque, de n'importe quel rang social, qui se voulait plus ou moins « indépendante » dans sa pensée et son mode de vie, ait surtout été vulnérable aux reproches masculins sur la question de sa sexualité. Selon les conventions d'alors quant au comportement féminin, Marguerite méritait certainement les reproches qu'elle reçut à ce propos, du fait de sa longue série d'aventures plus ou moins publiques (elle n'est guère ré-installée à Paris que son favori du moment, Saint-Julien, est tué devant sa porte ${ }^{4}$ ). Les critiques hostiles tendaient donc tout naturellement dans ce sens, souvent poussées par des motifs politiques et religieux à des outrances, telles que les accusations récurrentes d'inceste, qui remontent au moins jusqu'au protestant Reveille-matin des François (1574) ${ }^{5}$. Quant à Gournay, irréprochablemement chaste aux yeux de tous et généralement censée manquer d'attraits féminins, elle reçut plutôt le revers de la médaille et portait la péjorative étiquette de « vieille fille ».

\section{Gournay en dehors de la sexualité}

Avec le passage du temps, et d'une façon qui en révèle beaucoup sur la psychologie des locuteurs, l'identité publique de « vieille fille » que vivait Gournay est de plus en plus imbriquée dans un discours la qualifiant de vieille femme bizarre, de prétendue sibylle, voire de sorcière, comme dans un dialogue satirique de $1634:$ : Pucelle de mille ans, vieille Muse authentique $»^{6}$. Gournay fournit elle-même, dans l'Apologie, une analyse de cette transformation de femme savante en «épouventail », la suggestion de la sorcellerie s'y faisant sentir de façon remarquable :

Parmy nostre Vulgaire, on fagotte à fantasie l'image des femmes Lettrées : c'est à dire, on compose d'elle une fricassée d'extravagances \& de chimeres : \& dit-on en general, sans s'amuser aux exceptions ou distinctions, qu'elle sont jettées sur ce moule. [...] \& 
ne la voit-on plus, qu'avec des presomptions injurieuses, \& soubs la figure de cét épouventail. ${ }^{7}$

Ce passage en dit long sur la persistance dans l'histoire des images imposées aux femmes de leur vivant : tout comme Marguerite est restée «La Reine Margot », la reine lubrique selon le «mythe » que retrace Éliane Viennot, Gournay a continué pour une bonne partie de la critique moderne, jusqu'à des jours très récents, d'être assimilée au même stéréotype composite, avec, pour certain champions d'un Montaigne doué de présence éternelle, un élément de fille déloyale rajouté :

Avec son enthousiasme de vieille fille à cheveux blancs, $\mathrm{M}^{\text {lle }}$ de Gournay, qui eut le tort de vivre trop longtemps, paraissait un peu ridicule [...]; elle avait toujours été pédante, mais elle l'était devenue terriblement, et son attitude agressive ou grognon nuisait fort à son père. ${ }^{8}$

Les rôles de « vieille fille » et de «fille d'alliance » de Montaigne se réunissent dans ces paroles de Maurice Rat pour en faire sortir, ironiquement, une reconnaissance d'un pouvoir sexuel véritable, quoique négatif, dont Gournay à réussi à s'emparer par le moyen improbable du renoncement. C'est qu'elle s'est livrée en toute apparence d'humilité à l'autorité d'un «père » qui ne donnerait jamais sa «fille» en mariage.

Bien entendu, on s'est beaucoup penché récemment sur l'ambivalence de la relation imaginaire que Gournay a maintenue, à travers ses travaux éditoriaux ainsi que ses propres textes, avec son «père » absent. L'avantage défensif, même stratégique, de se constituer comme «ombre » a été analysé. Je souligne plutôt ici qu'un tel stratagème répond spécifiquement au fait qu'une femme intellectuelle, à la différence d'un homme, doive trouver des moyens de négocier sa sexualité avec le public, donc en public, et que cette nécessité lui impose un jeu qu'elle ne peut pas gagner à long terme, puisque il consiste paradoxalement à fabriquer pour ses adversaires les armes les plus aptes à se blesser, en se réduisant de femme intellectuelle en femme tout court. C'est un cercle vicieux : le fait que Gournay devienne «fille » garantit qu'elle deviendra « vieille fille».

\section{Marguerite dans les discours d'amour}

Le même processus se déroule du côté de Marguerite en termes contraires et fait de la femme une grande amoureuse. Dire simplement que les indiscrétions de son comportement fournissaient des munitions à ses ennemis ne tient pas compte d'une dynamique discursive dans laquelle elle était déjà inscrite sous le signe d'amour. Dans son cas, on peut remonter même au discours abondamment tenu à son sujet, dès sa petite enfance, par la poésie quasi-officielle de la cour ${ }^{9}$. Cependant Éliane Viennot fait remarquer qu'à 
partir des années 1570 , ce discours prend de plus en plus en compte ses activités intellectuelles et culturelles, et qu'en 1578 Guy Le Fèbvre de La Boderie la qualifie de l'incarnation du Parfait Amour « en des termes qui reprennent ceux dont elle aimait se parer ${ }^{10}$. Or ces termes appartenaient au néoplatonisme, adapté, comme on le sait, pour insister sur l'identité des sentiments amoureux et religieux («Car l'Amant, l'Aimé, l'Amour même / Qui est le Dieu unique en trois $»^{11}$ ), et l'occasion particulière du poème était la traduction de La Boderie du commentaire de Ficin sur le Banquet de Platon, traduction dédiée à la Reine de Navarre.

Si cette dernière « aimait se parer » de tel langage, ce n'était point par un simple goût de parure. C'est au fond une façon de valoriser la personne qu'elle est, ou voudrait être, étant donné le fait incontournable que, en tant que femme, il n'y a pas de définition discursive " pure » possible, c'est-àdire qui ne resterait pas rattachée à sa nature corporelle et sociale. Il s'agit d'une médiation idéalisante de sa féminité terrestre, comme moyen de réconcilier son identité corporelle avec ses aspirations intellectuelles. Cette médiation est obligatoire dans la mesure où c'est précisément en tant que femme qu'elle sera perçue lorsqu'elle va au-delà de son sexe. Ceci ne signifie pas du tout que le néoplatonisme de Marguerite manque de sincérité ou de sérieux. Au contraire, il est d'autant plus profond qu'il lui offre le seul rôle possible pour se justifier vis-à-vis des autres et d'elle-même : tout comme Gournay, elle se justifie, en s'emparant de ce discours amoureux qui, de toute façon, aurait été tourné contre elle - et qui fatalement le sera encore.

Bien avant 1578, Marguerite avait eu des amants indéniablement terrestres, notamment Louis Clermont d'Amboise, Seigneur de Bussy, qui est mort au cours de cette même année. Quelques épisodes de cette liaison sont assez ridicules, tels que l'occasion où Bussy dut sortir à la dérobée de sa chambre quand Henri de Navarre rentrait soudain de la chasse ${ }^{12}$; les circonstances de sa disparition furent d'ailleurs sordides et elles ne témoignent guère, non plus, d'une fidélité éternelle de la part de l'amant (de façon spectaculaire, on le sait bien, il tombe assassiné par les hommes du jaloux comte de Montsoreau, dont la femme était le nouvel objet des attentions de Bussy) ; toutefois, Marguerite en fit le deuil avec, pour ainsi dire, de grandes pompes néoplatoniciennes. Quelques « tombeaux » existent faisant allusion à leur relation comme à un amour éternel et en termes mythiques ${ }^{13}$. Plus révélateur encore, ainsi qu'évocateur, est le dialogue poétique «Ombre de Bussy », qui, que ce soit de Pibrac ou non, avait certainement son origine dans l'entourage de Marguerite et qu'elle classait parmi ses poèmes préférés ${ }^{14}$. Je m'intéresse moins au contenu assez banal de ces textes qu'à la gestion discursive qu'ils opèrent de cette liaison sexuelle illicite afin de la spiritua- 
liser, et avec un tel succès que leur grand amour est entré dans le mythe de Marguerite comme renouvelé au début du XVIII' siècle ${ }^{15}$. Dans l' «Ombre », où l'esprit de «Lysis » assassiné apparaît à « Flore » pour faire ses adieux, le maître mot « vertu » sert à unir la force morale et la force physique, âme et corps, féminin et masculin. La dame affligée déclare "Puisque la Vertu seule, en aimant, je poursuis, / Peu me chaut que chascun fondre en larmes me voie »; l'ombre attribue son meurtre à la jalousie des autres de «l'esclat de ma vertu», alors que le poète fait reproche à la cour corrompue dans les mêmes termes : «O Prince malheureux, / Tu estains la vertu pour avancer le vice ! 16 .

La liaison avec Bussy sert de cas exemplaire d'une pratique discursive de Marguerite assez connue, et qu'on peut tracer, par exemple, dans ce qui reste de sa correspondance avec Jacques de Harlay, seigneur de Chanvallon, le galant successeur de Bussy, avec qui, d'après Éliane Viennot, « elle va tenter de vivre la passion supérieure dont elle rêve, selon le modèle néoplatonicien qu'elle affectionne ${ }^{17}$. D'une façon ou d'une autre, il est toujours question de son positionnement vis-à-vis de « l'Amour [ . . .] ce dieu [. . .] qui possède ma raison ${ }^{18}$. Mais ce modèle reste fragile, précaire, fait dont l'humour qui fait partie intégrante de son discours amoureux épistolaire la montre peut-être consciente. Éliane Viennot a beau prendre son recours à l'imaginaire comme une stratégie pour éviter la « chute » qui consiste en « la fin de la passion ${ }^{19}$, sur ce point biographique elle est essentiellement d'accord avec le propos d'Yves Cazaux, qui s'approche du même phénomène de l'autre côté : « [e]lle avait besoin de s'exalter pour aimer » ${ }^{20}$. Mais ce besoin n'est pas antérieur au discours qui en découle ; il témoigne plutôt de la pré-inscription de Marguerite dans un discours plus large de féminité qu'elle essaye de maîtriser, de rendre sien, pour qu'il ne puisse être utilisé contre elle. Elle est en un sens obligée de devenir adepte de néoplatonisme pour éviter de devenir putain.

Pourtant, c'est bien ce qu'elle devient, bien entendu, dans nombreuses attaques, dont la plus vulgaire et la plus vicieuse connue est sans doute le Divorce satyrique, ce pamphlet d'origine inconnue et d'orientation politique indéfinie ${ }^{21}$, qui date de l'époque du rétablissement de Marguerite à Paris (entre 1605 et 1610). Ce qui est certain c'est que le roi Henri IV y est également visé, car l'ensemble du discours, y compris des remarques peu flatteuses à l'égard de sa propre moralité et de sa religion, est censé sortir de sa bouche. Il va sans dire que les aventures de Marguerite sont alors rigoureusement dénuées de toute prétention néoplatonicienne. Voilà, entre autres, le grand amour avec Bussy recyclé en farce du point de vue du cocu royal plus ou moins complaisant : 
Aussi je ne fus point tellement aveuglé en un faict si sensible \& si apparent, que je ne apperceusse clairement comme les autres que Clermont [c.-à-d., Bussy] maintefois la baissoit toute en juppe sur la porte de sa chambre, tandis que le soir pour luy donner loisir de se mettre au lict, je jouois ou me promenois avec ma Noblesse dans la salle. ${ }^{22}$

Justement, le grand intérêt du pamphlet pour nous consiste en sa fonction de réécriture consciente, sa préoccupation non seulement de la basse réalité de l'histoire amoureuse de Marguerite mais aussi de sa représentation discursive ; c'est par le biais d'un personnage déjà investi dans son propre langage que l'auteur s'en prend à elle :

Jusques icy ses fautes n'estoient que fleurs quoy qu'assez mal converts, l'âge, le temps, $\&$ sa volontaire prison d'Usson en faisoient tolerer, excuser, \& cacher quelques uns, son habitude au mal avoit lassé les langues les plus babillardes, \& sa longue absence avoit desja faict oublier son nom parmy les grands, mais pour couronner son œuvre \& donner la derniere main à ce discours de sa vie, elle a voulu revenir voir la France, \& n'a pas moins voulu chercher, que Paris, les yeux de la Cour, pour servir de theatre $\&$ de tesmoins à son Histoire, $\&$ telle qu'elle promet d'estre cy apres. ${ }^{23}$

Ce biais explique le besoin de démolir l'édifice discursif que le néoplatonisme construit pour souder corps et esprit, extérieur et intérieur, beauté et vertu. Ce n'est pas seulement que le corps de Marguerite est devenu monstrueux mais aussi qu'il va à l'encontre des valeurs morales, tout en corrompant le discours même : " l'huile de jasmin dont elle oingt chaque nuict le corps ne peut [...] empescher la puante odeur de sa reputation ${ }^{24}$. Quant à la vertu, terme récurrent dans le discours néoplatonisant autour de la liaison de Marguerite et Bussy, la seule prononciation du mot doit produire en elle une réaction corporelle violente :

entendre proferer sans rougir ny penser qu'on se mocque d'elle, ces mots, honneur \& vertu, qui sont opposez \& directement ennemis de sa profession. [. . .] \& s'est rendue sujette à ne pouvoir tolerer qu'on tousse, ou qu'on parle en sa presence, tant le soupçon $\&$ le mespris d'elle mesme luy faict aprehender le discours de ses actions. ${ }^{25}$

Si Le Divorce satyrique effectue une inversion implicite du discours dans lequel Marguerite s'était déjà inscrite, une autre satire, La Ruelle mal assortie, procède en juxtaposant ce discours et son contraire. Il s'agit d'un dialogue censé avoir lieu entre elle et son amant, qui est physiquement beau mais bête et pas intéressé du tout par la philosophie, pour en dire le moins. La docte dame lui prêche le néoplatonisme en vain pour finalement céder à la passion physique. Éliane Viennot a mis hors de doute, dans un article magistral, la possibilité que l'auteur de ce dialogue soit Marguerite ellemême ${ }^{26}$. Cependant, il y a peut-être lieu de tempérer son jugement sur le ton négatif, dans la mesure où même l'humour grivois de la satire fait écho, de façon caricaturale, à un humour raffiné chez Marguerite qui lui aussi rend 
compte de la tension, sinon de l'opposition, entre les aspects physiques et spirituels de l'amour. Dans cette même mesure, la Ruelle s'en prend à Marguerite, non seulement, comme Le Divorce satyrique, par le biais de sa pré-inscription dans le discours d'amour, mais par le biais de sa propre reconnaissance de cette pré-inscription.

Néanmoins, la Ruelle reste un dialogue qui revendique, de façon franchement structurale, une incompatibilité entre les prétentions philosophiques de Marguerite et sa pratique amoureuse. Théoriquement, il resterait encore un moyen discursif possible pour gérer cette dichotomie, à savoir en termes de synthèse, une solution qui ne serait pas, bien entendu, adaptée à des fins satiriques, puisqu'elle refléterait le but visé par le néoplatonisme même. C'est une solution qu'on peut trouver notamment dans quelques textes contemporains du néoplatonisme anglais, tels que des poèmes de John Donne et la pièce de Shakespeare sur Antoine et Cléopâtre. Ce sont des textes où l'humour, même un humour très sexualisé, joue un rôle médiateur mais, peut-être, affirmatif, en insistant sur le fait incontournable que le physique et le spirituel, le bas et le haut, sont essentiellement liés dans la nature humaine.

C'est également la perspective, du moins on peut le soutenir, d'une comédie shakespearienne qui met sur scène précisément Marguerite de Valois et dont le titre, Les Peines d'amour perdues, servirait très bien pour exprimer, satiriquement ou pas, la thématique en question. Comme on le sait bien, le couple amoureux principal y est constitué par le roi de Navarre et la princesse de France ; la pièce fait référence, bien que de façon imprécise, à la visite de Marguerite à son mari en 1578 et aux activités intellectuelles associées à la cour de Nérac. L'aspect satirique de cette pièce prend pour cible, non pas les femmes en général, encore moins la princesse, mais les aspirations irréalistes des hommes, qui ont l'idée de s'adonner à la philosophie à l'exclusion de l'amour. En même temps, y compris du côté des femmes, qui n'en restent pas moins chastes et intelligentes, on y trouve un discours sexuel constant, tenu à travers des jeux de mots et sous-entendus, qu'on peut qualifier des plus obscènes chez Shakespeare ; jusqu' au point de provoquer la seule objection soulevée dans son œuvre contre la grossièreté elle-même, quand une dame d'honneur fait remarquer à un courtisan, de façon passagère, «Comme, come, you talk greasily, your lips grow foul ${ }^{27}$. Malgré cette remarque, la pièce semblerait vouloir mettre en accord la pensée et la vie amoureuses de Marguerite, à la différence, par exemple, du récit de Scipion Dupleix dans son Histoire de Henry III, qui choisit le même moment historique, où les aventures amoureuses et de Henri de Navarre et de sa femme étaient particulièrement flagrantes, pour son sommaire du caractère de la dernière. Ayant dûment loué ses qualités de grande princesse, 
Dupleix lui reproche des faits, et des paroles, trop orduriers pour être décrits : " ie rougirois si ie couchois sur le papier ce que ie luy en ay oui dire serieusement à elle-mesme $»^{28}$.

De toute évidence, accorder le noble et l'obscène relève du royaume des souhaits exaucés, c'est-à-dire de la comédie. Dans la tragédie on pourrait s'attendre, sous la pression « réaliste » du genre, à l'instabilité d'une synthèse idéalisée entre le haut et le bas, à moins que les confins mêmes du genre ne cèdent pour créer un nouvel espace imaginaire. C'est justement ce dernier effet qu'on peut discerner dans Antoine et Cléopâtre. La situation est plus ambiguë, mais encore plus pertinente, dans une pièce de George Chapman qui mérite d'être rapprochée du personnage de Marguerite de Valois.

Bussy d'Amboise ne mentionne pas Marguerite. Cependant, la partie de la vie du héros mise sur scène dans cette tragédie sanglante et romanesque trace sa carrière à partir de son introduction à la cour par Monsieur (François-Hercule, duc d'Anjou), événement qui a eu lieu en 1575, et jusqu'à son assassinat par les hommes de Montsoreau quatre ans après. Comme d'habitude sur la scène élisabéthaine, le déroulement de l'action est fort comprimé, de sorte qu'on a l'impression que Bussy rencontre la comtesse de Montsoreau immédiatement à la cour et que leurs amours, avec leurs consequences fatales pour lui, s'amorcent tout de suite. Or Bussy n'était pas été nécessairement un personnage inconnu du public anglais, même au début du XVII ${ }^{\mathrm{e}}$ siècle, car il avait joué un rôle prééminent dans les négociations concernant l'éventuel mariage entre Monsieur et la reine Élisabeth. On aurait donc bien pu remarquer la substitution qu'effectue le dramaturge : c'était avec Marguerite que Bussy était entré en relation à ce moment-là.

Chapman encourage peut-être cette observation par un changement de nom : sa comtesse, en réalité Françoise, est rebaptisée Tamyra (nom fort improbable pour une française et qui est en plus, fortuitement ou pas, un anagramme partiel de «Marguerite »). Plus significatif encore, le mariage entre Montsoreau et sa femme se termine par un divorce, ce qui ne fut pas le cas en réalité (au contraire, le couple passa le restant de sa vie ensemble en harmonie, ayant au moins six enfants). Ce divorce est représenté comme imposé par l'honneur du mari blessé et à sa plus grande douleur : " I do forgive thee, and upon my knees / (With hands held up to heaven) wish that mine honour / Would suffer reconcilement to my love. »(V.iii.247-4929). C'est un tableau pathétique qui chevauche, non seulement un aspect du discours autour du divorce de Henri IV et de Marguerite ${ }^{30}$, aspect repris de façon exagérée dans le Divorce satyrique, mais aussi le «mythe » qui voulait que la « réclusion [de Marguerite] à Usson » en Auvergne pour des raisons politiques a été «motivée par les dérèglements de sa vie amoureuse » ${ }^{31}$. Or Brantôme fait référence à son exil «parmi les déserts, rochers et montagnes 
d'Auvergne $»^{32}$, tandis que la femme adultère chez Chapman prétend « to the open deserts / (Like to hunted tigers) I will fly: / Eating my heart, shunning the steps of men »(V.iii.243-45). Brantôme exprime aussi son espoir que, malgré le fait que « [n]ostre reyne Marguerite est ainsin un peu en division et divorce aveq le roy son mary », néanmoins Dieu « les metra un jour en bonne union, en despit du temps misérable », union figurée en termes néoplatoniciens comme « et de corps et d'ame et d'amityé, comme ils ont estez $»^{33}$. Plus tard, Brantôme a rajouté une note marginale à son manuscrit: «Dieu a voulu despuys qu'ils se sont bien réconciliez ${ }^{34}$. On serait tenté d'en identifier l'occasion comme le rétablissement de Marguerite à Paris en 1605, événement qui, d'après L'Estoile, « réveillèrent les esprits curieux, et fournirent d'ample matière de discours à toutes sortes de personnes ${ }^{35}$. Figurait parmi les personnes ainsi concernées certainement l'auteur du Divorce satyrique, mais peut-être aussi G. Chapman, dont la pièce en question peut bien dater de cette année ${ }^{36}$.

De notre point de vue, ce qui est plus important que ces indices assez spécifiques, c'est que la relation amoureuse entre les personnages Bussy et Tamyra est dépeinte dans des termes qui n'ont rien à voir avec la liaison notoirement terre à terre qui entraîna la mort de Bussy mais qui puisent de façon insistante dans la philosophie néoplatonicienne. D'une part, cela reflète une tendance généralisée à travers l'œuvre de Chapman, aussi bien dans sa poésie que dans ses pièces de théâtre. D'autre part, les échos du discours filtrant le deuil de Marguerite sont particulièrement forts. Le terme central dans le néoplatonisme de la pièce, et surtout le maître mot de Bussy, est celui de « vertu », qui d'ailleurs porte chez Chapman sa connotation de force à la fois morale et physique, comme dans le discours autour du Bussy historique. Et comme dans ce discours, cette qualité, où amalgame de qualités, est censée transcender la moralité conventionnelle. Ainsi Bussy rassure Tamyra, qui se condamne moralement par anticipation et pense, pour ainsi dire, à sa «puante [. . .] reputation » («I fly my sex, my virtue, my renown [...] / See, see the gulf is opening, that will swallow / Me and my fame for ever » [II.ii.173-75]), en lui répondant que non seulement il va maintenir la «fame » de sa maîtresse par le moyen de son propre «value » (III.i.36, 38) mais qu'il va « make it fly out of the mouths of Kings / In golden vapours, and with awful wings » (40-41), ce qui n'est guère le cas dans le Divorce satyrique. Et à la fin, le seul représentant de la religion, l'ombre du moine tué, prévoit rien moins que l'apothéose de l'âme du héros, dont l'assassinat constitue une crucifixion symbolique à l'instar du martyre de Lysis :

Farewell brave relicts of a complete man :

Look up and see thy spirit made a star,

Join flames with Hercules : and when thou set'st 
Thy radiant forehead in the firmament,

Make the vast continent, crack'd with thy receipt,

Spread to a world of fire : and th'aged sky,

Cheer with new sparks of old humanity. (V.iii.268-74)

La représentation de cette liaison amoureuse chez Chapman reste pour autant fort ambiguë, ce qui a justement provoqué des interprétations de la pièce divergentes. Car la rhétorique de «vertu »/《vertue » et de transcendance risque de sembler être greffée sur une affaire de chair et d'os. Il est assez clair, en ses propres paroles, que Tamyra n'éprouve que du désir sexuel, même si celui-ci est irrésistible et dangereux. Bussy, quant à lui, s'adonne à l'aventure amoureuse comme à un véhicule d'affirmation de soi au mépris d'autrui, avec l'énergie violente et aggressive qui caractérise toutes ses démarches. De ce point de vue, et étant donné la tendance de l'action à dégénérer vers des effets mélodramatiques, le cadre philosophique se révèle artificiel, surtout que le personnage du moine qui s'en porte garant peut se lire finalement comme un simple entremetteur. En somme, l'idéal d'une synthèse entre le haut et le bas se raréfie, se fragilise jusqu'au point où il se révèle précisément comme n'étant qu'une fonction discursive. Cet effet, on peut le soutenir, s'étend à l'identité d'une Marguerite dans les coulisses : elle est la prisonnière d'un discours amoureux qu'elle prétendait justement manipuler.

\section{Conclusion}

Est-ce qu'on ne peut pas dire, finalement, que Marie de Gournay, tout en rejetant ce discours, en devient pareillement prisonnière ? Sa carrière de « fille »/《 vieille fille», poursuivie à la fois avec acharnement et avec un sens de la fatalité, nécessite que son néoplatonisme soit purgé de l'élément érotique et afin d'en valoriser une version permettant l'union intellectuelle de « grands esprits » à travers les barrières du sexe et du temps, c'est-à-dire de la mort. Or cette purgation n'est pas sans laisser de traces dans son œuvre, lesquelles comportent une puissante ambivalence affective. Ses éloges de femmes intellectuelles (Hypathia, Cornelia), de femmes saintes (Deborah, Catherine, Marie Madeleine) ou encore de femmes guerrières (les Amazones, Jeanne d'Arc) ont tous en commun l'exaltation d'une force qui pourrait être masculine, et qui est donc androgyne. L'amour, pour elle, doit devenir cette qualité mystique et asexuée que Diotime, prêtresse légendaire, aurait apprise à Socrate. Il est clair que pour Gournay, tout ce qui a rapport au corps impose sur les femmes la faiblesse, la vulnérabilité. Cet élément humain est régulièrement rejeté sur les persécutions sociales subies par des femmes, sur la bêtise des femmes qui essaient de séduire des hommes indignes ou qui se 
laissent séduire, surtout quand il s'agit d'attraits superficiels (le rang, les richesses).

Mais au fond de tout cela se trouve, pour ainsi dire, une des rare choses que Marie de Gournay a dites une fois pour toutes, et toute jeune, dans sa seule œuvre de fiction. Le Promenoir de Monsieur de Montaigne raconte, bien sûr, la tragédie d'une jeune princesse, donc d'une femme ayant théoriquement le potentiel d'exercer du pouvoir, la capacité de l'indépendance, qui réagit à son statut d'objet d'échange entre hommes dans un monde patriarcal, en cédant abjectement et aveuglément à son propre côté érotique, plutôt que de rester la fille de son père. Cela se termine mal, moins à cause des perfidies des hommes (bien qu'il y en ait beaucoup) qu'à cause de la force de l'éros même, qui s'impose au niveau du corps (Alinda subit une grossesse, puis une mort physiquement grotesque), et en fonction du temps (son mari se lasse d'elle). Chez Gournay, ceux qui vivent un grand amour sexualisé ne gagnent qu'une épitaphe renforçant le gouffre entre le bas et le haut imposé par l'amour même : «L'amour, Tyran au monde, est Dieu dans l'Helisée ${ }^{37}$.

Ceci représente, peut-être, une vérité si profonde et troublante pour Gournay qu'elle a effacé l'épitaphe, avec plusieurs autres éléments parfois dits « féministes », des les dernières éditions de cet ouvrage très populaire. Mais cette vérité serait profonde et troublante, non pas à cause de l'avertissement ainsi offert implicitement aux jeunes femmes, lequel après tout est parfaitement conventionnel et impeccablement moral, mais plutôt parce qu'elle insiste sur l'infranchissabilité de ce que le néoplatonisme, même celui de Gournay, existe pour franchir, puisqu'il s'agit en fin de compte d'une question de vie et de mort. L'érotique reste intrinsèquement partie de la nature humaine, donc mortelle ; le rejeter revient à reconnaître son emprise. Quant à la femme écrivain ou femme intellectuelle à la Renaissance, pour autant qu'elle pourrait se faire transporter dans son imaginaire aux Champs Élysées, le vécu de Marguerite, ainsi que celui de Gournay, laisse entendre l'impossibilité d'échapper à la tyrannie discursive de l'Amour. Le dieu installé dans l'au-delà est « toujours déjà » atteint de cette tyrannie.

Université François-Rabelais, Tours

\section{Notes}

1. Éliane Viennot, Marguerite de Valois. Histoire d'une femme, histoire d'un mythe, Paris, Payot et Rivages, 1993, p. 219 ; Marjorie Henry Ilsley, A Daughter of the Renaissance. Marie le Jars de Gournay. Her Life and Works, La Haye, Mouton, 1963, p. 100.

2. Voir Ilsley, p. 97-100. 
3. Ibid., p. 99.

4. Sur cette affaire, voir Viennot, Marguerite de Valois, p. 208-11.

5. Nicolas Barnaud (?), Le Reveille-Matin des François et de levrs voisins: Composé par Eusebe Philadelphe Cosmopolite, en forme de Dialogues, «Edinburgh: Jaques James », c.-à-d. Strasbourg, Bernard Jobin, 1574, fac-similé édition, Paris, Éditions d'Histoire Sociale, 1977 : «Monsieur son frere scauoit bien qu'il auoit eu son pucellage » (Dialogue 1, p. 44).

6. Voir Ilsley, p. 236. Cf. Mario Schiff, La Fille d'alliance de Montaigne, Marie de Gournay, 1910, ré-impression Genève, Slatkine, 1978, p. 23, qui attribue l'accusation de sorcellerie au fait que Gournay a pratiqué l'alchimie pendant un certain temps.

7. Nous citons Les Advis, Ou, Les Presens de la Demoiselle de Gournay (1641), p. 605[A], dans l'édition de Jean-Philippe Beaulieu et Hannah Fournier, 3 vol., Amsterdam et Atlanta, Georgia, Rodopi, 1997-, t. 2, p. 297.

8. Maurice Rat, introduction à son édition des Essais de Montaigne, 2 vol., Paris, Garnier, 1962, t. 1, p. xiii.

9. Par exemple, le sonnet liminaire de La Charite de Pierre Ronsard s'adresse « À l'unique perle, Marguerite de France, Royne de Navarre » (Euvres complètes, éd. Jean Céard, Daniel Ménager et Michel Simonin, 2 vol., Paris, Gallimard, Bibliothèque de la Pléiade, 1993, t. 1, p. 577) ; dans le poème principal elle est représentée comme composée de diverses fleurs (v. 74) ; dans «L'Amour Amoureux » elle est la « [p]erle et fleur des François » (v. 28). Sur le « paradigme Marguerite-Fleur-Perle » voir Catherine Magnien-Simonin, Marguerite de France Reine de Navarre et son temps, Actes du Colloque d'Agen (12-13 octobre 1991) organisé par la Société française des seizièmistes et le Centre Matteo Bandello d'Agen, ed. Madeleine Lazard et J. Cubelier de Beynac, Agen, Centre Mattheo Bandello, 1994, p. 146.

10. Viennot, Marguerite de Valois, p. 235.

11. Cité ibid.

12. Léo Mouton, Bussy d'Amboise et Madame de Montsoreau. D'après des documents inédits, Paris, Hachette, 1912, p. 94 ; Pierre Chevallier, Henri III. Roi shakespearien, Paris, Fayard, 1985, p. 297.

13. Voir Pierre de Bourdeille, abbé de Brantôme, Euvres complètes, éd. Ludovic Lalanne, 11 vol., Paris, La Société de l'Histoire de France, 1864-82, t. 5, p. 192 et note 1.

14. Voir Viennot, Marguerite de Valois, p. 120.

15. Ce renouvellement est marqué par l'édition des Mémoires en 1713 (éd. J. Godefroy, Liège, J. F. Broncart) accompagnée d'autres textes dans un « ensemble [qui] [. . .] lie résolument Marguerite à Bussy » (Viennot, Marguerite de Valois, p. 298). Le fait d'y avoir inclus une version du dialogue fort néoplatonisant La Fortune de la Cour, où Bussy figure comme personnage, témoigne de l'encradrement philosophique opéré par ce recueil. Les Mémoires eux-mêmes, bien sûr, ne font pas mention de cette liaison, mais ils parlent de Bussy de façon très admirative, et Scipion Dupleix, entre autres, trouve que Marguerite y dissimule mal ses sentiments ; il suggère en plus que l'amour de Marguerite a porté le roi son frère à autoriser l'assassinat (Histoire de Henry III, Roy de France et de Pologne, Paris, Claude Sonnius, 1630, p. 111-12). Par contre, que les récits de Dupleix servaient les intérêts diffamatoires du cardinal de Richelieu a été bien établi par Éliane Viennot (Marguerite de Valois, p. 264-67). 
16. Nous citons le texte dans Pierre de l'Estoile, Registre-Journal du regne de Henri III, éd. Madeleine Lazard et Gilbert Schrenck, 4 vol., Genève, Droz, 1992-2000, t. 3, p. 39-40.

17. Viennot, Marguerite de Valois, p. 133.

18. Marguerite de Valois, Correspondance 1569-1614, éd. Éliane Viennot, Paris, H. Champion, 1998, lettre 115, p. 173-74.

19. Viennot, Marguerite de Valois, p. 149.

20. Mémoires et autres écrits de Marguerite de Valois, la reine Margot, éd. Yves Cazaux, Paris, Mercure de France, 1986, p. 22.

21. Sur ces questions, voir Viennot, Marguerite de Valois, p. 246, et Claude-Gilbert Dubois, «Le Divorce Satyrique de la Reyne Marguerite », in Marguerite de France Reine de Navarre et son temps, p. 99-106.

22. Divorce satyrique, En forme de Factum pour \& au nom du Roy Henry IV, etc., in Recueil de diverses pieces servans à l'histoire de Henry III, Cologne, Pierre de Marteau, [1663], p. 235-58, p. 239.

23. Ibid., p. 253-54.

24. Ibid., p. 258.

25. Ibid., p. 253.

26. Éliane Viennot, «Marguerite de Valois et La Ruelle mal assortie : une attribution erronée », Nouvelle Revue du Seizième Siècle, no 10, 1992, p. 81-98.

27. William Shakespeare, Love's Labour's Lost, in The Riverside Shakespeare, éd. sous la direction de G. Blakemore Evans et J. J. M. Tobin, $2^{\mathrm{e}}$ éd., Boston, Houghton Mifflin, 1997, IV.i.137. Pour une discussion plus complète du phénomène discursif, voir notre livre, Self-Speaking in medieval and early modern English drama. Subjectivity, discourse and the stage, Houndmills, Basingstoke, Macmillan/New York, St. Martin's, 1997, p. 246-64.

28. Dupleix, Histoire de Henry III, p. 100-101.

29. Nous citons George Chapman, Bussy d'Ambois [sic], éd. N. S. Brooke, The Revels Plays, Manchester, Manchester University Press, 1964.

30. À témoin les paroles attribuées au roi larmoyant par Dupleix dans son Histoire de Henri $I V$, mais dont l'authenticité est fort douteuse, selon Charles Merki, La reine Margot et la fin des Valois (1553-1615). D'après les mémoires et les documents, Paris, PlonNourrit, 1905, p. 401 :

Ah, la malheureuse [. . .] elle sait bien que je l'ay toujours aimée et honorée, et elle point moy, et que ses mauvais déportements nous on fait séparer, il y a longtemps, l'un de l'autre.

31. Viennot, Marguerite de Valois, p. 262.

32. Brantôme, op. cit., t. 8, p. 56.

33. Ibid., t. 8, p. 57-58.

34. Ibid., t. 8, p. 57 , note 2.

35. Pierre de L'Estoile, Journal de l'Estoile pour le règne de Henry IV, 3 vol., t. 2 (1601-1609), éd. André Martin, 4e éd., Paris, Gallimard, 1958, p. 170. 
128 / Renaissance and Reformation / Renaissance et Réforme

36. On propose généralement 1604 ou 1605 comme la date de composition ; la première édition en était publiée en 1607.

37. Marie de Gournay, Le Promenoir de Monsieur de Montaigne: Texte de 1641, avec les variantes des éditions de 1594, 1595, 1598, 1599, 1607, 1623, 1626, 1627, 1634, éd. Jean-Claude Arnould, Études Montaignistes 26, Paris, H. Champion, 1996, p. 168, note 15. 